\title{
Laparoscopy Endoscopy Cooperative Surgery for Inflammatory Fibroid Polyp in the Esophagus
}

\author{
Kohei Oka ${ }^{1,2}$, Ken Inoue ${ }^{3}$, Naoto Iwai ${ }^{2}$, Tasuku Hara ${ }^{2}$, Yutaka Inada ${ }^{2}$, Toshifumi Tsuji ${ }^{2}$, \\ Sosuke Komiyama ${ }^{4}$, Takashi Okuda ${ }^{2}$, Soujin Sai ${ }^{5}$, Akihiro Nagata ${ }^{6}$, Toshiyuki Komaki ${ }^{2}$, \\ Yuji Naito $^{3}$, Yoshito Itoh $^{3}$ and Keizo Kagawa ${ }^{2}$
}

\begin{abstract}
:
Inflammatory fibroid polyp (IFP) appears most often in the stomach. We herein report an extremely rare case of esophageal IFP resected using laparoscopy endoscopy cooperative surgery (LECS). A 73-year-old man with dysphagia underwent esophagogastroduodenoscopy. A 50-mm semi-pedunculated submucosal tumor was observed in the esophagogastric junction. Endoscopic ultrasonography showed a homogeneous, hypoechoic, and well-vascularized tumor in the second and third layers of the stomach, but endoscopic ultrasonography-guided fine-needle aspiration failed to establish a definite diagnosis. Since he was suffering from dysphagia, LECS was performed. The resected specimen proved that the tumor was an IFP originating from the esophagus.
\end{abstract}

Key words: inflammatory fibroid polyp (IFP), esophagus, laparoscopy endoscopy cooperative surgery (LECS)

(Intern Med 58: 2357-2362, 2019)

(DOI: 10.2169/internalmedicine.2595-18)

\section{Introduction}

Inflammatory fibroid polyp (IFP) appears commonly in the stomach, small intestine, and colon and is caused by stimuli to the affected area. We experienced a rare case of IFP originating in the esophagus and attempted its excision using a minimally invasive surgical procedure: laparoscopy endoscopy cooperative surgery (LECS).

\section{Case Report}

A 73-year-old man presented to our hospital with progressive dysphagia. He also had a history of black stool. He had taken some analgesics for a few weeks because of lower back pain, but no antacids had been prescribed. Laboratory tests showed microcytic anemia (red blood cell count 204x
$10^{4} / \mu \mathrm{L}$, hemoglobin $5.4 \mathrm{~g} / \mathrm{dL}$, mean corpuscular volume 88.2 fL). Esophagogastroduodenoscopy, which was performed due to suspected upper gastrointestinal bleeding, revealed a semi-pedunculated 50-mm submucosal tumor (SMT) in the fundus of the stomach. It appeared to originate from the oral side of the esophagogastric junction prolapsing into the stomach. There were some erosions on its surface, but no active bleeding was observed. Contrast-enhanced computed tomography showed a low-enhancement tumor in the same location. Endoscopic ultrasonography (EUS) demonstrated a homogeneous, hypoechoic, and well-vascularized tumor in the second and third layer of the stomach (Fig. 1). However, it was difficult to demonstrate sequentiality to the adjacent layer because of the tumor prolapse. Although endoscopic ultrasonography-guided fine-needle aspiration (EUS-FNA) was performed twice for the pathological evaluation and some interstitial tissues were obtained, they failed to provide

\footnotetext{
${ }^{1}$ Department of Gastroenterology and Hepatology, North Medical Center, Kyoto Prefectural University of Medicine, Japan, ${ }^{2}$ Department of Gastroenterology and Hepatology, Fukuchiyama City Hospital, Japan, ${ }^{3}$ Department of Gastroenterology and Hepatology, Kyoto Prefectural University of Medicine, Japan, ${ }^{4}$ Department of Surgery, Osaka General Hospital of West Japan Railway Company, Japan, ${ }^{5}$ Department of Surgery, Fukuchiyama City Hospital, Japan and ${ }^{6}$ Department of Pathology, Fukuchiyama City Hospital, Japan Received: December 28, 2018; Accepted: February 25, 2019; Advance Publication by J-STAGE: May 22, 2019 Correspondence to Dr. Ken Inoue, keninoue71@koto.kpu-m.ac.jp
} 

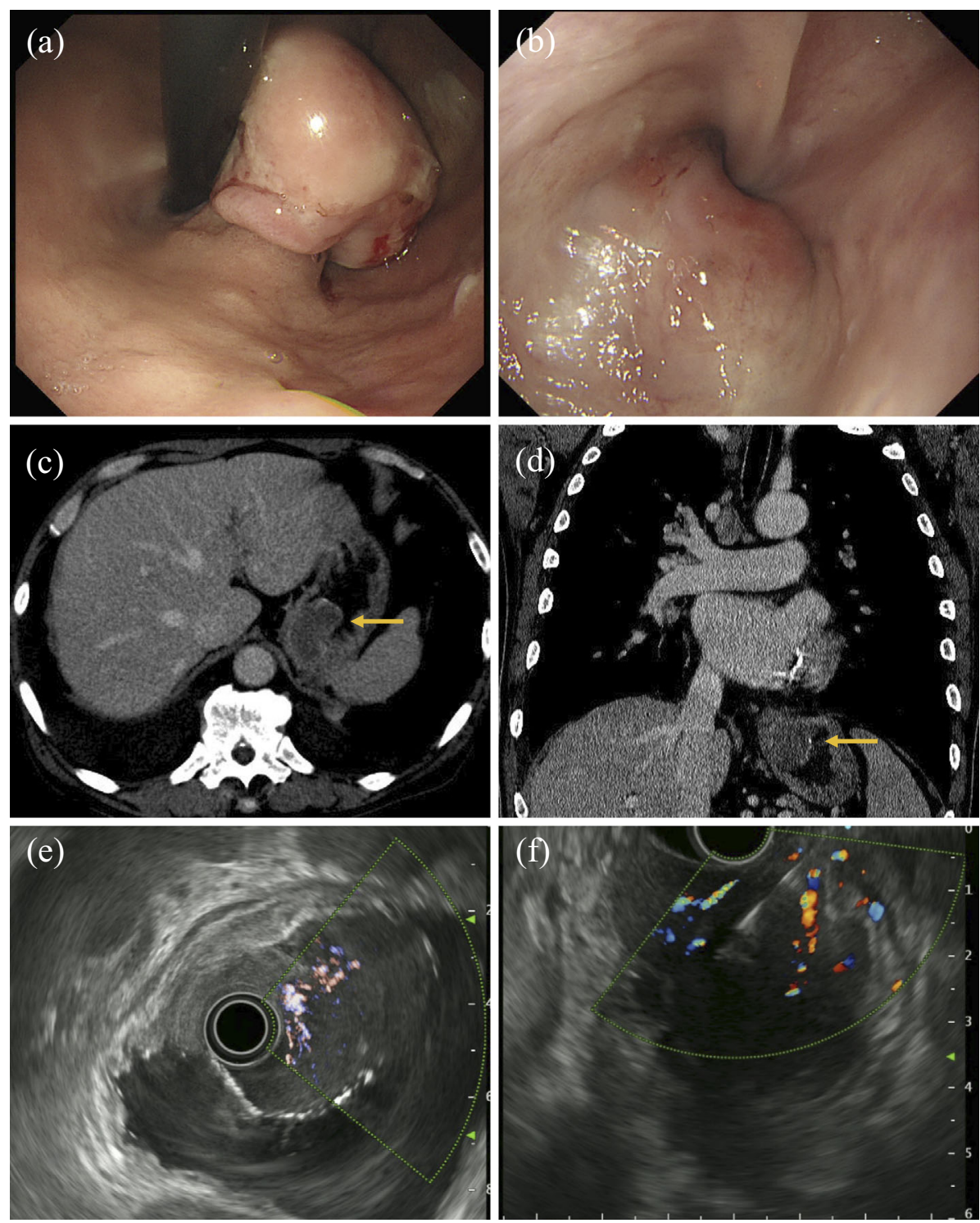

Figure 1. (a) On upper gastrointestinal endoscopy, a submucosal tumor was seen in the fundus. (b) The tumor was semi-pedunculated and seemed to originate from the esophagogastric junction. (c, d) Computed tomography showed a low-enhanced tumor (arrow). (e) Endoscopic ultrasonography showed a hypoechoic and well-vascularized tumor in the third layer of the stomach. (f) Punctures of fine-needle aspiration were performed.

a definite diagnosis due to an insufficient sample amount. The collected tissues were negative for c-kit, DOG1, and desmin; the lesion was therefore suspected of being a benign tumor, like leiomyoma.

Since the patient had been suffering from dysphagia, surgical excision was scheduled. At the request of the patient and his family, LECS was performed as an alternative to proximal gastrectomy. We attempted circumferential dissection with the support of laparoscopy. After we marked around the lesion with a needle knife, we administered a submucosal injection of sodium hyaluronate $0.4 \%$ (MucoUp; Boston Scientific, Tokyo, Japan), following which a precut was made, and dissection of the lesion from the oral end with scissors forceps (ClutchCutter; Fujifilm, Tokyo, Japan) was performed. Due to severe submucosal fibrosis, we continued the circumferential dissection from the anal end.
Even though some injuries of the lamina muscularis occurred due to severe fibrosis, the wall defect was closed immediately using the laparoscopic hand-sewn technique. Finally, the tumor was completely resected without major procedural complications (Fig. 2).

A histopathological examination revealed fibroblastic spindle cells with eosinophilic infiltration, concentrically arranged around small vessels. The tumor was also covered with stratified squamous epithelium of the esophagus, not with the lining epithelium of the stomach (Fig. 3). The tumor size was $50 \mathrm{~mm}$, and a sufficient margin was secured. The tumor invaded the deep submucosal layer. It was difficult to detect the precise depth because the boundary was obscure; however, the tumor had invaded to almost the final third of the submucosal layer. The final diagnosis was an IFP of the esophagus. The patient has not complained of 

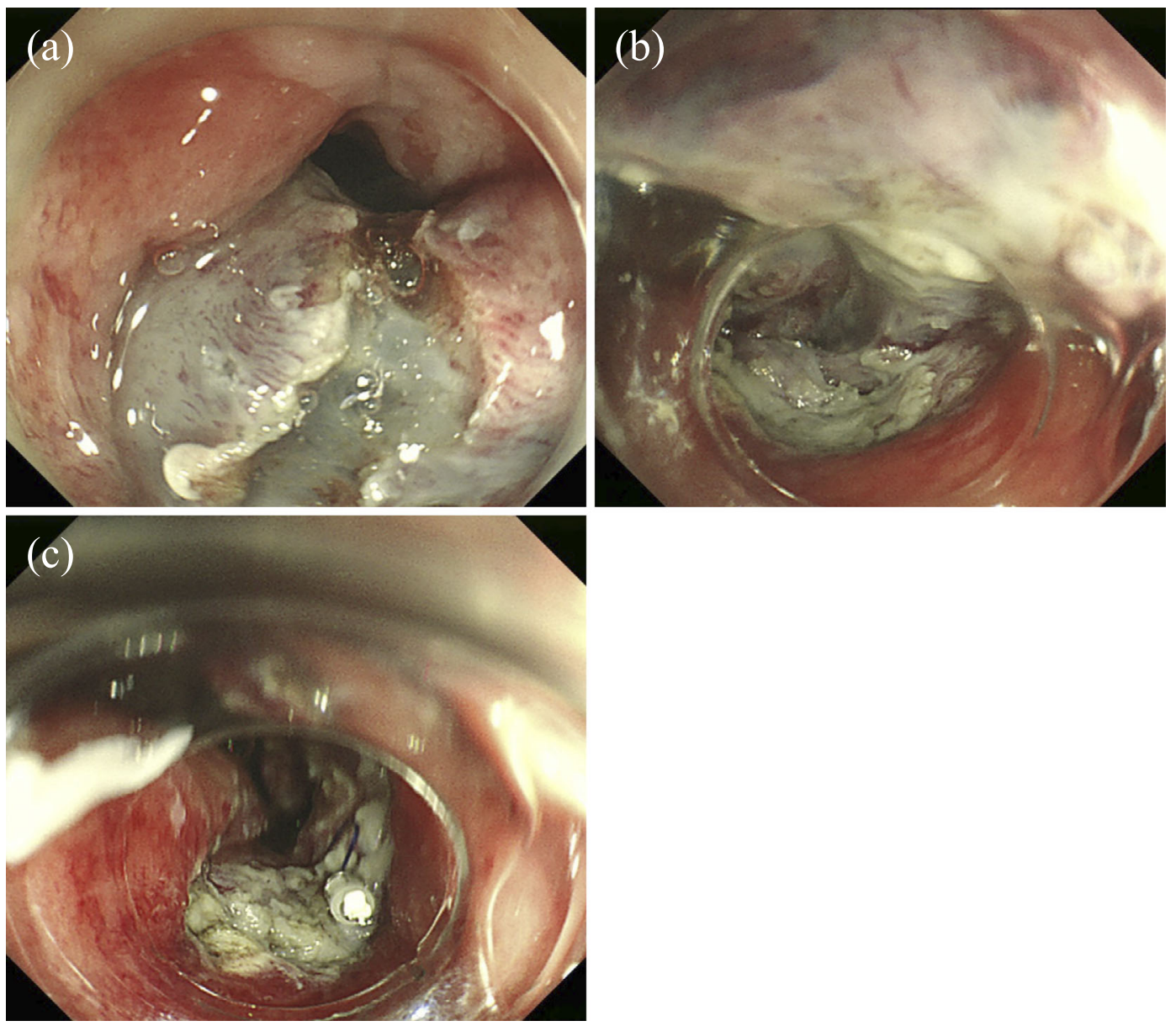

Figure 2. Laparoscopy endoscopy cooperative surgery. (a) Dissection of the lesion was initially started from the oral end. (b) Due to severe submucosal fibrosis, circumferential dissection was attempted from the anal end. (c) Some injuries of the lamina muscularis were seen due to severe fibrosis, but the wall defect was closed immediately using the laparoscopic hand-sewn technique.

any symptoms since the surgery, and there have been no signs of recurrence in the year since the resection (Fig. 4).

\section{Discussion}

An IFP is an inflammatory polyp that arises in the submucosa and mucosa of the gastrointestinal tract. Although it has generally been considered as a reactive and nonneoplastic lesion, its pathogenesis remains controversial. Some recent reports have revealed that IFP harbors plateletderived growth factor receptor A mutations, which may attribute a neoplastic nature (1). IFP sometimes has erosions or ulcers on its surface, which are suspected of being the cause of microcytic anemia, as in our case. IFP is microscopically characterized by fibroblastic spindle cells and eosinophilic infiltration. Spindle cells appear concentrically arranged around the blood vessels, which is often referred to as an "onion-skin like appearance." Immunohistochemistry has shown that the spindle cells in IFP are positive for CD34 but negative for CD117 and protein S-100 (2). In our case, the resected specimen showed typical pathological findings consistent with IFP; therefore, an immunohistochemical analysis was not performed.
IFPs commonly originate in the stomach and less frequently in the small intestine and colon, but only a few reports have described its presence in the esophagus (3). A literature search on PubMed with the key words "inflammatory fibroid polyp" and "esophagus," produced 10 case reports describing esophageal IFP with detailed clinical information (Table) (3-12). The mean tumor size was $77 \mathrm{~mm}$ (range: 13-130 mm). Dysphagia was the most common presentation. Most of the lesions originated from the lower thoracic esophagus or the esophagogastric junction. Endoscopic ultrasonography was performed in three cases, which revealed homogeneous and hypoechoic tumors. In addition, in one detailed case, the tumor was found to be well vascularized, similar to our case. Among these cases, eight underwent surgical excision without recurrence. In the other two cases, endoscopic mucosal resection (EMR) was performed, but both of them developed local recurrences within a year after EMR $(6,8)$. To our knowledge, this was the first case of LECS being used to resect esophageal IFP, although it has been attempted in some cases of gastric IFP (13).

The preoperative diagnosis in our case was leiomyoma because the lesion was hypoechoic and existed in the same layer as the submucosa, according to the EUS survey, and 

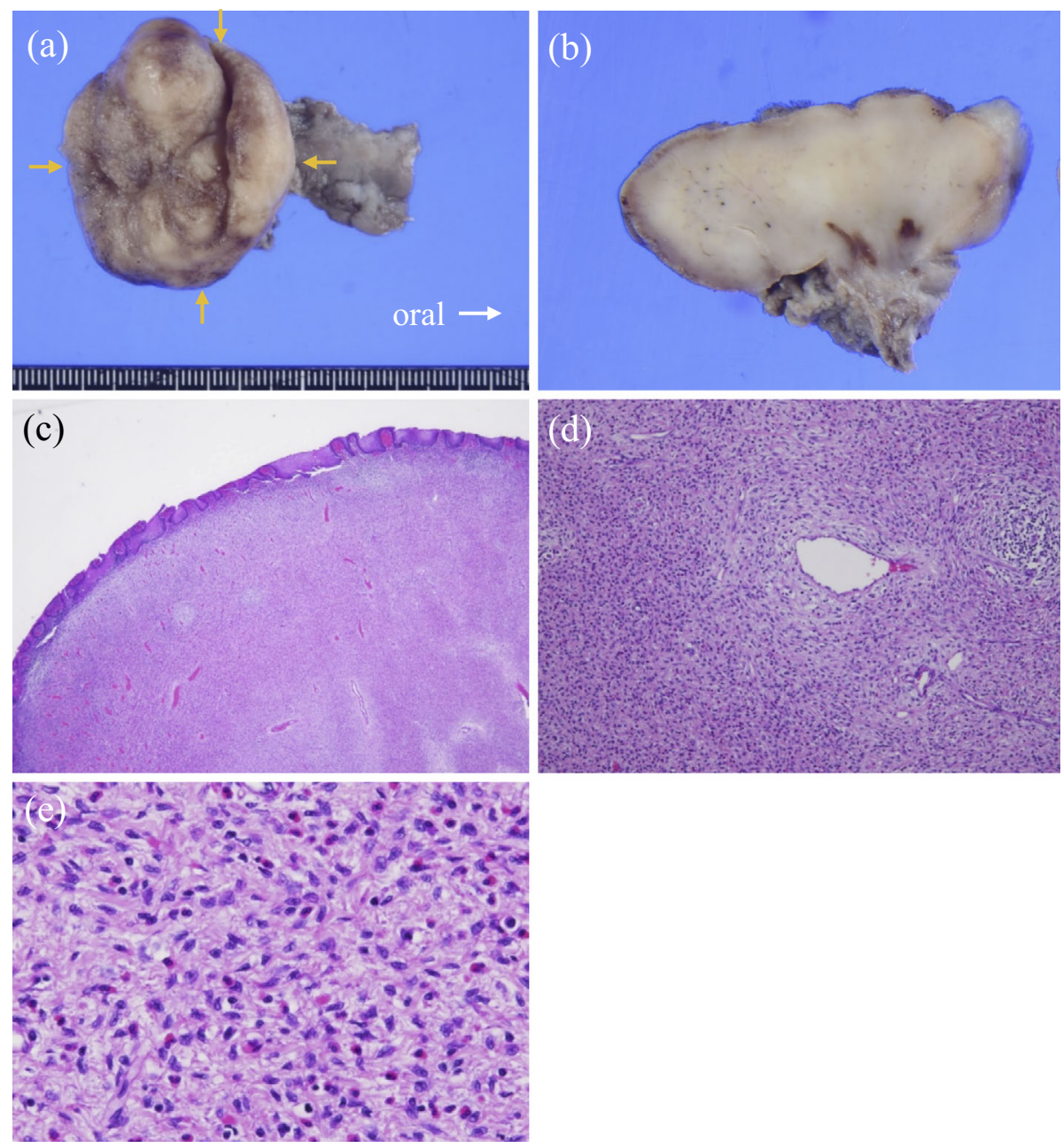

Figure 3. (a) Macroscopic appearance of the resected specimen, measuring $50 \mathrm{~mm}$. (b) Sectional image of the resected specimen. (c, d, e) Hematoxylin and Eosin staining sections (magnification, 4x, $10 \times, 40 \times$, respectively). The tumor was covered with squamous epithelium of the esophagus. Submucosal spindle cells with eosinophilic infiltration around blood vessels - "onion skin appearance" - were observed.
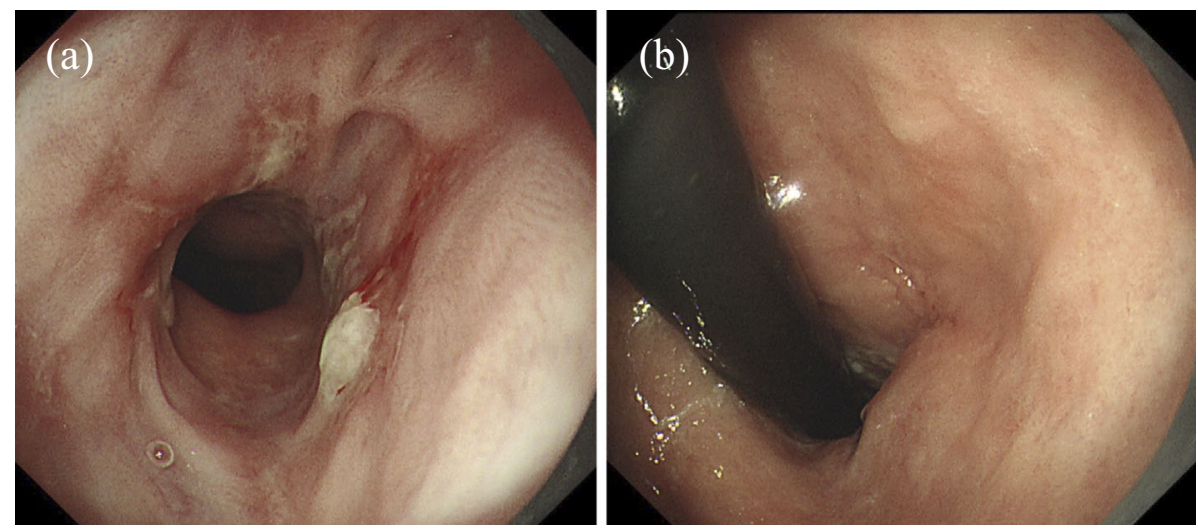

Figure 4. Endoscopic images recorded one year after resection. (a, b) The images show some reflux esophagitis but no tumor recurrence.

no malignant tissues were obtained by EUS-FNA. Other differential diagnoses were gastrointestinal stromal tumor, schwannoma, and inflammatory myofibroblastic tumor (2). However, such differentiation is challenging because these submucosal tumors show a similar hypoechoic pattern on EUS, and a sufficient amount of submucosal tissues is required to achieve a definite pathological diagnosis. In addition, pedunculated tumors are usually difficult to diagnose 
Table. Case Reports Describing Esophageal IFP.

\begin{tabular}{|c|c|c|c|c|c|c|c|c|}
\hline Reference & Age & Sex & Size $(\mathrm{mm})$ & Portion & EUS & Symptoms & Treatment & Recurrence \\
\hline 3 & 60 & M & 60 & EGJ & NA & - & Surgery & NA \\
\hline 4 & 76 & $\mathrm{~F}$ & NA & $\mathrm{Lt}$ & + & $\begin{array}{c}\text { Dysphagia } \\
\text { +epigastric pain }\end{array}$ & Surgery & NA \\
\hline 5 & 58 & M & NA & $\mathrm{Lt}$ & NA & Pyrosis & Surgery & - \\
\hline 6 & 48 & M & 20 & EGJ & + & $\begin{array}{c}\text { Dysphagia } \\
\text { +epigastric pain }\end{array}$ & EMR & + \\
\hline 7 & 76 & M & 90 & EGJ & NA & $\begin{array}{c}\text { Dysphagia } \\
\text { +epigastric pain }\end{array}$ & Surgery & - \\
\hline 8 & 25 & M & 13 & EGJ & + & Pyrosis & EMR & + \\
\hline 9 & 33 & M & 110 & $\mathrm{Lt}$ & - & NA & Surgery & NA \\
\hline 10 & 69 & M & 70 & $\mathrm{Lt}$ & - & Chest pain & Surgery & - \\
\hline 11 & 59 & M & 120 & Ut & NA & Dysphagia & Surgery & NA \\
\hline 12 & NA & NA & 130 & $\mathrm{Lt}$ & NA & NA & Surgery & NA \\
\hline Presented case & 73 & M & 50 & EGJ & + & Dysphagia & LECS & - \\
\hline
\end{tabular}

EGJ: esophagogastric junction, EMR: endoscopic mucosal resection, EUS: endoscopic ultrasonography, IFP: inflammatory fibroid polyp, LECS: laparoscopy endoscopy cooperative surgery, Lt: lower thoracic esophagus, NA: not applicable, Ut: upper thoracic esophagus

by EUS. We should have attempted to perform the EUSguided investigation from the opposite side (oral side).

IFP is usually excised with either endoscopy or surgery, depending on the lesion's size and location. While a small IFP may be resected using EMR, there is a possibility of bleeding and local recurrence. In contrast, surgical excision of IFP in the esophagogastric junction is usually performed by proximal gastrectomy. There have been many attempts to minimize surgical invasiveness. Laparoscopic proximal gastrectomy is one of the less-invasive approaches; nevertheless, it may cause anastomosis-related complications, like reflux symptoms (14). In contrast, LECS has often been performed for SMTs in the stomach. It is generally less invasive than conventional surgery and does not cause anastomosis, although it may cause cicatricial stenosis. Despite the seromuscular layer being artificially perforated in classical LECS, we attempted circumferential dissection by endoscopic submucosal dissection with the support of laparoscopy because the tumor location made it difficult to anastomose the esophagus and stomach after full layer dissection with classical LECS.

\section{Conclusion}

To our knowledge, this is the first report of a case of an IFP of the esophagus resected by LECS. When diagnosing SMT of the esophagus, IFP should be considered as a differential diagnosis. Evaluations of more cases of IFP will be needed in order to clarify the characteristics of this disease.

\section{The authors state that they have no Conflict of Interest (COI).}

\section{Acknowledgement}

We are grateful to our patient, who cooperated with the publication of this report.

\section{References}

1. Schildhaus HU, Cavlar T, Binot E, Buttner R, Wardelmann E, Merkelbach-Bruse S. Inflammatory fibroid polyps harbour mutations in the platelet-derived growth factor receptor alpha (PDGFRA) gene. J Pathol 216: 176-182, 2008.

2. Liu TC, Lin MT, Montgomery EA, Singhi AD. Inflammatory fibroid polyps of the gastrointestinal tract: spectrum of clinical, morphologic, and immunohistochemistry features. Am J Surg Pathol 37: 586-592, 2013.

3. Bosch O, Gonzalez Campos C, Jurado A, et al. Esophageal inflammatory fibroid polyp. Endoscopic and radiologic features. Dig Dis Sci 39: 2561-2566, 1994.

4. Costa PM, Marques A, Távora I, Oliveira E, Diaz M. Inflammatory fibroid polyp of the esophagus. Dis Esophagus 13: 75-79, 2000.

5. Solito B, Anselmino M, Tognetti A, Vignati S, Rossi M. Rare case of inflammatory fibrous polyp of the esophagus. Dis Esophagus 15: 326-329, 2002.

6. Zinkiewicz K, Zgodzinski W, Dabrowski A, Szumilo J, Cwik G, Wallner G. Recurrent inflammatory fibroid polyp of cardia: a case report. World J Gastroenterol 10: 767-768, 2004.

7. Godey SK, Diggory RTJWJoSO. Inflammatory fibroid polyp of the oesophagus. World J Surg Oncol 3: 30, 2005.

8. Yamane $T$, Uchiyama $K$, Ishii $T$, et al. Case of inflammatory fibroid polyp of the esophagogastric junction. Dig Endosc 21: 97100, 2009.

9. Tudose I, Andrei F, Calu V, Staniceanu F, Miron A. Giant inflammatory fibroid polyp. Rom J Intern Med 50: 179-185, 2012.

10. Modi C, Shah A, Depasquale JR, Shah N, Spira RS. A large prolapsed inflammatory fibroid polyp of the esophagus: an unusual presentation. Gastroenterol Hepatol (NY) 9: 322-325, 2013.

11. Rawashdeh B, Meyer M, Gill J, Moslemi M, Kim S. Unusual presentation of a giant benign inflammatory polyp in the upper esophagus. Int J Surg Case Rep 6C: 206-209, 2014.

12. Vincze B, Mezei P, Hanyik J, et al. A giant inflammatory fibroid polyp of the esophagus. Magy Seb 70: 69-73, 2017 (in Hungarian).

13. Hajer J, Havluj L, Whitley A, Gurlich R. Non-exposure endoscopic-laparoscopic cooperative surgery for stomach tumors: first experience from the Czech Republic. Clin Endosc 51: 167173, 2018. 
14. Kosuga T, Ichikawa D, Komatsu S, et al. Feasibility and nutritional benefits of laparoscopic proximal gastrectomy for early gastric cancer in the upper stomach. Ann Surg Oncol 22 (Suppl 3): S929-S935, 2015.
The Internal Medicine is an Open Access journal distributed under the Creative Commons Attribution-NonCommercial-NoDerivatives 4.0 International License. To view the details of this license, please visit (https://creativecommons.org/licenses/ by-nc-nd/4.0/).

(C) 2019 The Japanese Society of Internal Medicine Intern Med 58: 2357-2362, 2019 